\title{
New Type of High-Frequency Discrete VLF Emissions (“BIRDS”) at Auroral Latitudes
}

\author{
J. Manninen ${ }^{1}$, T. Turunen ${ }^{1}$, N.G. Kleimenova ${ }^{2}$, M.J. Rycroft ${ }^{3}$, L.I. Gromova ${ }^{4}$ \\ ${ }^{1}$ Sodankyla Geophysical Observatory, Sodankyla, Finland \\ ${ }^{2}$ Schmidt Institute of the Physics of the Earth RAS, Moscow, Russia \\ ${ }^{3}$ CAESAR Consultancy, Cambridge, UK \\ ${ }^{4}$ Pushkov Institute of Terrestrial Magnetism, lonosphere, and Radiowave Propagation \\ RAS, Troitsk, Moscow
}

E mail (jyrki.manninen@sgo.fi).

Accepted: 26 January 2018

\begin{abstract}
Based on VLF observations in Northern Finland at Kannuslehto (KAN, $L \sim 5.5$ ), recently we revealed a new and previously unknown daytime type of VLF emissions at frequencies well above $4 \div 5 \mathrm{kHz}$. These emissions have neither been seen before because they were hidden by strong impulsive sferics originating in lightning. The peculiar VLF signals, discovered after filtering out the sferics, were studied. It is shown here that these emissions, which have a complicated spectral structure, occur during the winter around local noon under the quiet solar and geomagnetic conditions (Vsw $<400 \div 500 \mathrm{~km} / \mathrm{s}, \mathrm{B}_{\mathrm{IMF}}<5 \div 7 \mathrm{nT}, \mathrm{Np}<5 \div 8 \mathrm{~cm}^{-3}, A \mathrm{E}<150 \div 200 \mathrm{nT}$ ). These emissions are usually right-hand polarized and can last for several hours as a series of separate short discrete wideband (from 4 to $10 \mathrm{kHz}$ and higher) signals, each with a duration between one and several minutes. We suppose that these waves are generated via the electron-cyclotron resonance mechanism deep inside the magnetosphere at much lower L-values than KAN. However, the details of the generation and propagation mechanisms of these newly discovered VLF emissions remain unclear.
\end{abstract}

(c) 2018 BBSCS RN SWS. All rights reserved

Keywords: whistler-mode VLF emissions, solar wind - magnetosphere interactions, wave-particle interaction, physics of the magnetosphere

\section{Introduction}

The natural whistler-mode electromagnetic waves at audio frequencies are called Very Low Frequency (VLF) emissions (Helliwell, 1965). They are common wave phenomena in the Earth's environment. These VLF waves are a very fundamental subject in the magnetospheric plasma physics due to their ability to efficiently interact with energetic electrons in the magnetosphere via a resonant interaction between the waves and energetic electrons inside the magnetosphere (e.g., Trakhtengerts and Rycroft, 2008). The VLF emissions propagate in the whistler-mode away from their source near the equatorial region in the magnetosphere and are guided to the ionosphere by density gradients that extend along the geomagnetic field lines. Theoretically (Smith et al., 1960) and experimentally (Carpenter, 1968), it was found that the ducted propagation of VLF waves is only possible at frequencies that are lower than half of the equatorial electron gyrofrequency $\left(f_{\mathrm{ce}}\right)$ of the given L-shell.

Our study is based on the VLF observations in Northern Finland at Kannuslehto station (KAN, L 5.5, geographic coordinates $\left.67.74^{\circ} \mathrm{N}, 26.27^{\circ} \mathrm{E}\right)$. The VLF emissions are recorded digitally in the frequency band of $0.2 \div 39 \mathrm{kHz}$ by two orthogonal magnetic loop antennas oriented in the North-South and East-West directions. So, we can calculate the horizontal angles of wave arrival but with a $180^{\circ}$ uncertainty, because there is no vertical electrical antenna. The description of this equipment is given in Manninen (2005).
The equatorial electron frequency $\left(f_{\mathrm{He}}\right)$ in the magnetosphere at $L \sim 5 \div 6$ is on the order of $\sim 4 \div 6 \mathrm{kHz}$, so, the guided whistler-mode waves, generated in the magnetosphere in this area, can reach the Earth's surface at the auroral latitudes at frequencies not higher than $\sim 3.0 \mathrm{kHz}$. Really, as a rule, the most typical natural VLF emissions observed at KAN are widely known chorus, hiss and different kinds of quasi-periodic emissions at frequency less than $\sim 4 \div 5 \mathrm{kHz}$.

However, many naturally occurring VLF waves at higher frequencies (above $4 \div 6 \mathrm{kHz}$ ) could not be studied due to strong atmospherics (sferics) originating in lightning discharges and hiding all other waves (e.g., Ohya et al., 2015).

To study the natural high-frequency VLF waves, we have to develop and apply special digital programs, which filter out the strong impulsive sferics by a broadband digital filter (from 0.6 to $16 \mathrm{kHz}$ ), with properly rounded edges so as not to cause deleterious effects on the dynamic spectral analysis. Over successive intervals of $20 \mathrm{~ms}$ (the duration of the filter) the signal is reduced to zero, and corrections are applied to the remaining power estimate for the power loss occurring during these 20 ms intervals. A correction is then applied for the small power loss of the dynamic spectral signal. Of course, weak sferics are still seen in VLF dynamic spectra, because the number of sferics is about 40 per second, and if we filter them all there is nothing left. Usually, we allow the data loss of $15 \div 20 \%$, which does not delete magnetospheric VLF events. Remaining horizontal lines are not PLHRs, but signals originated from Russian Zevs navigation transmitter located at Kola Peninsula. 


\section{Dec 2013}
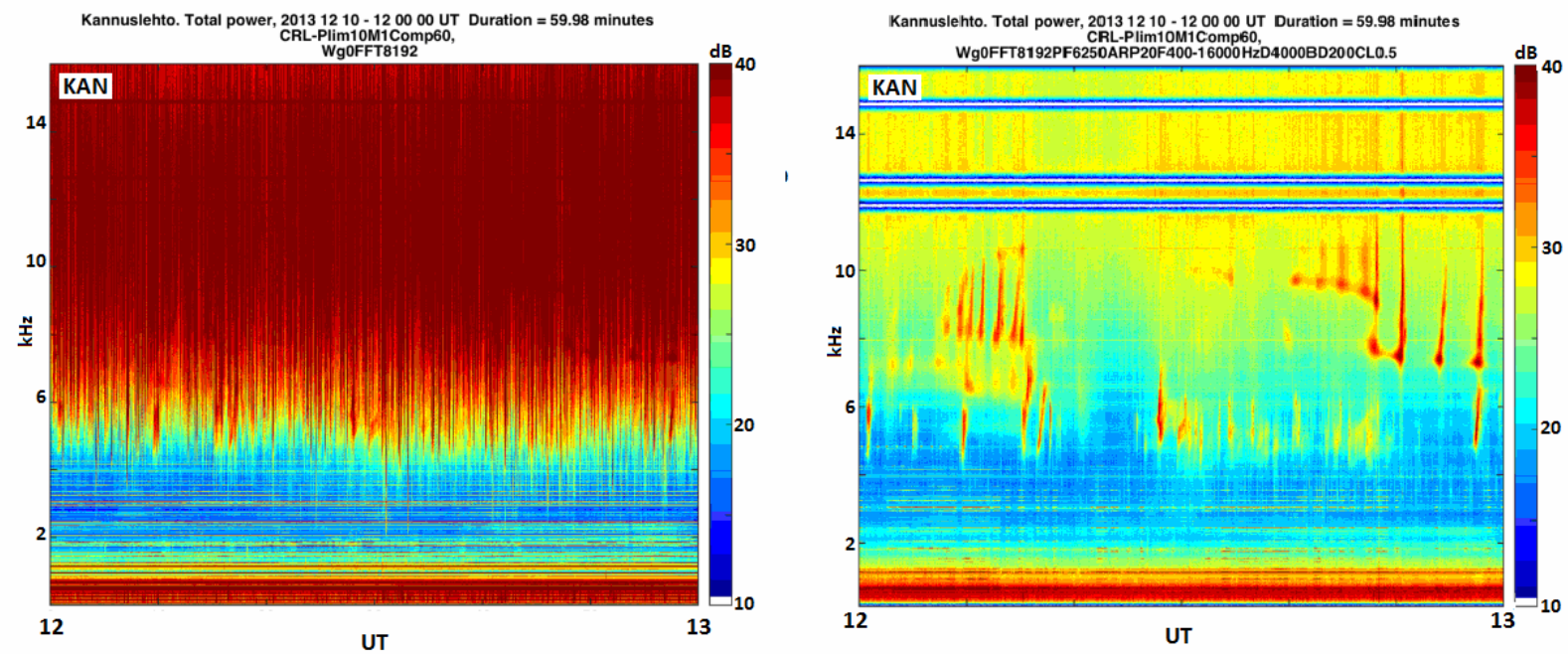

Fig. 1. (a) - An example of the initial raw spectrogram without any filtering, (b) - the same data after filtering out the sferics and PLHRs. Three high-frequency blue horizontal lines - removed radio transmitter traces.

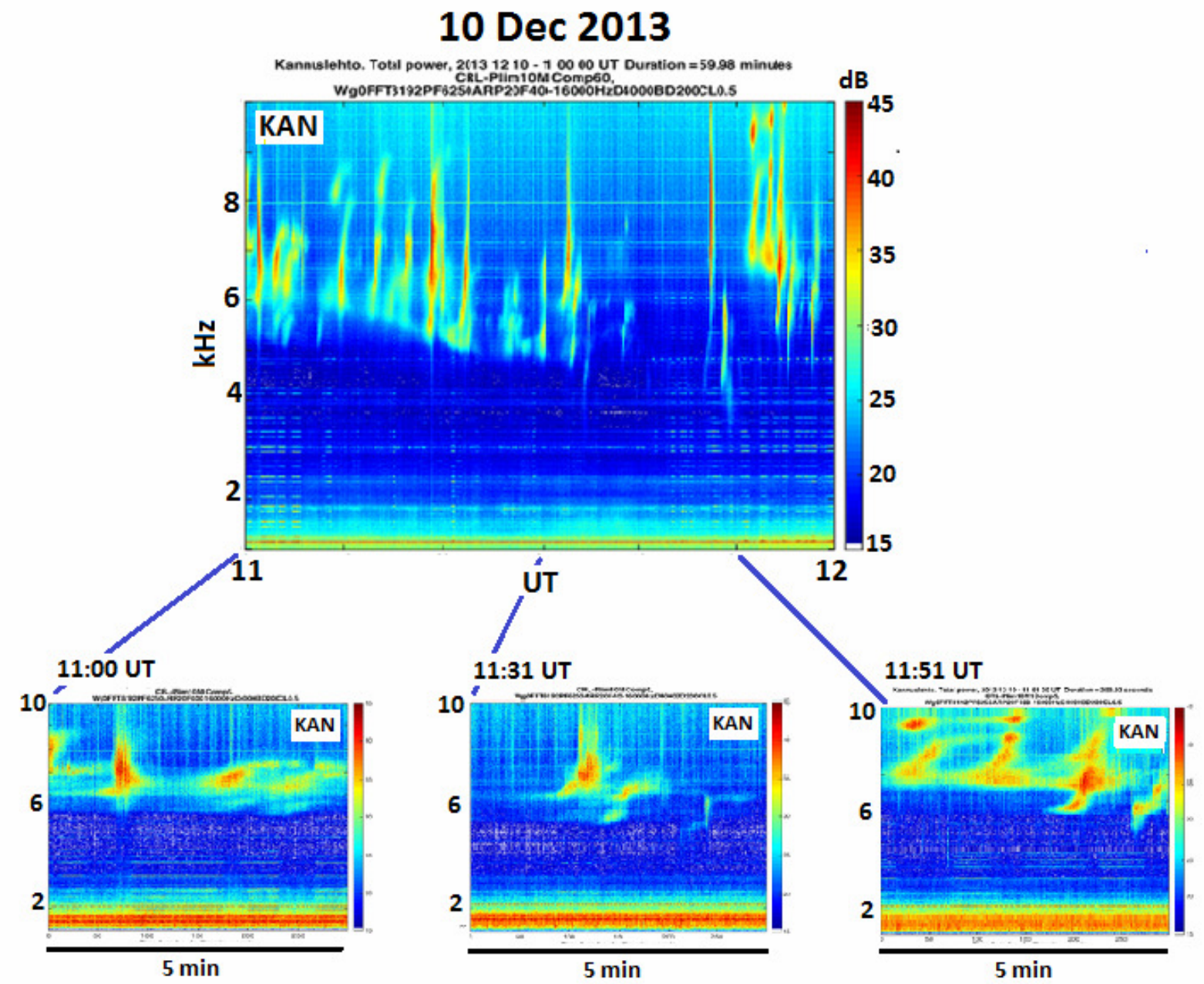

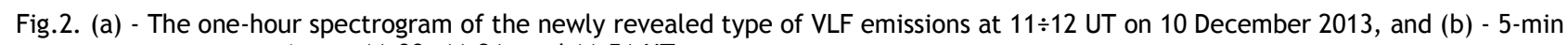
spectrograms starting at $11.00,11.31$, and 11.51 UT 

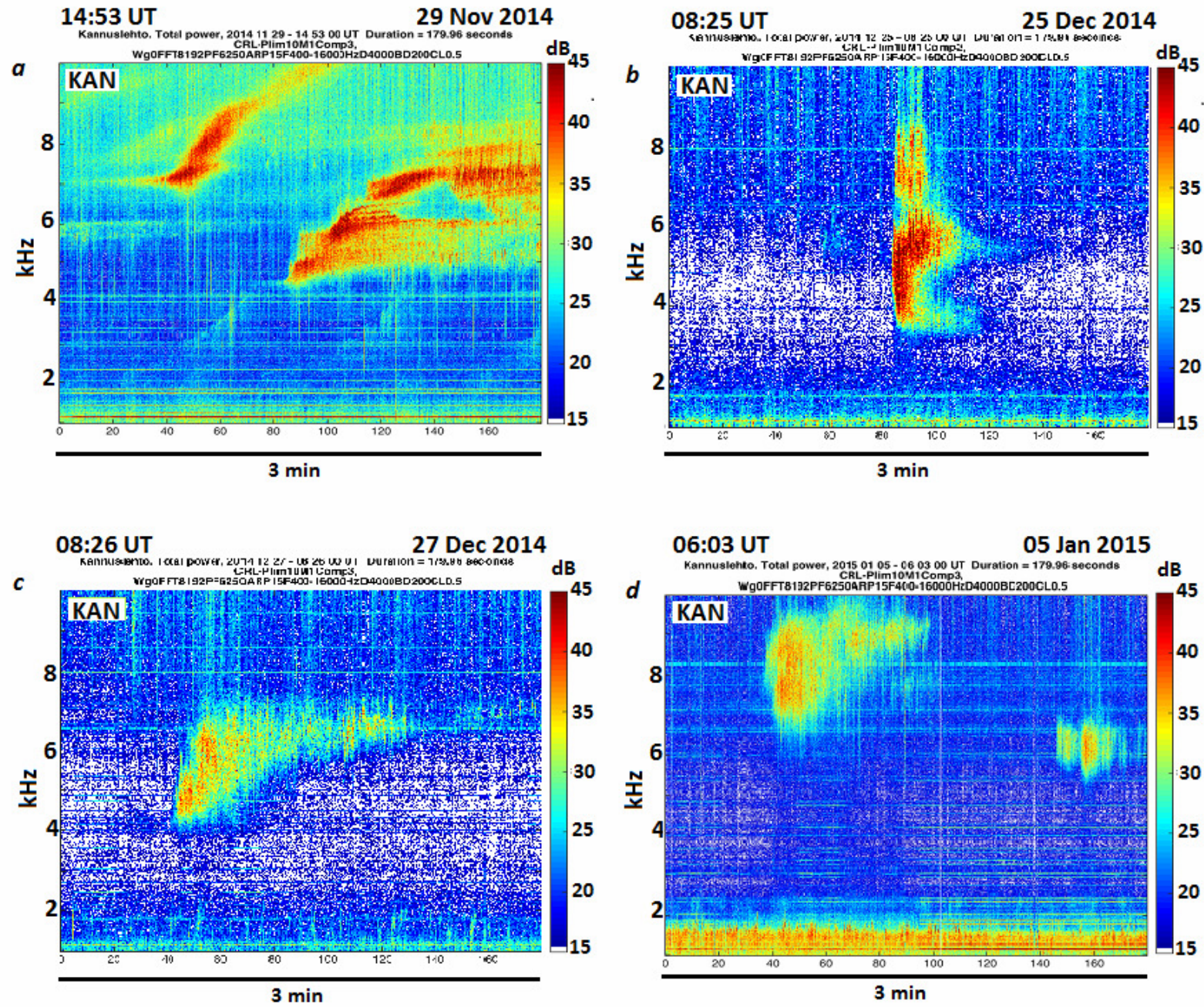

Fig.3. Examples of 3-min VLF spectrograms demonstrating different dynamic spectra of the newly revealed type of VLF emissions.

\section{Nov 2014}
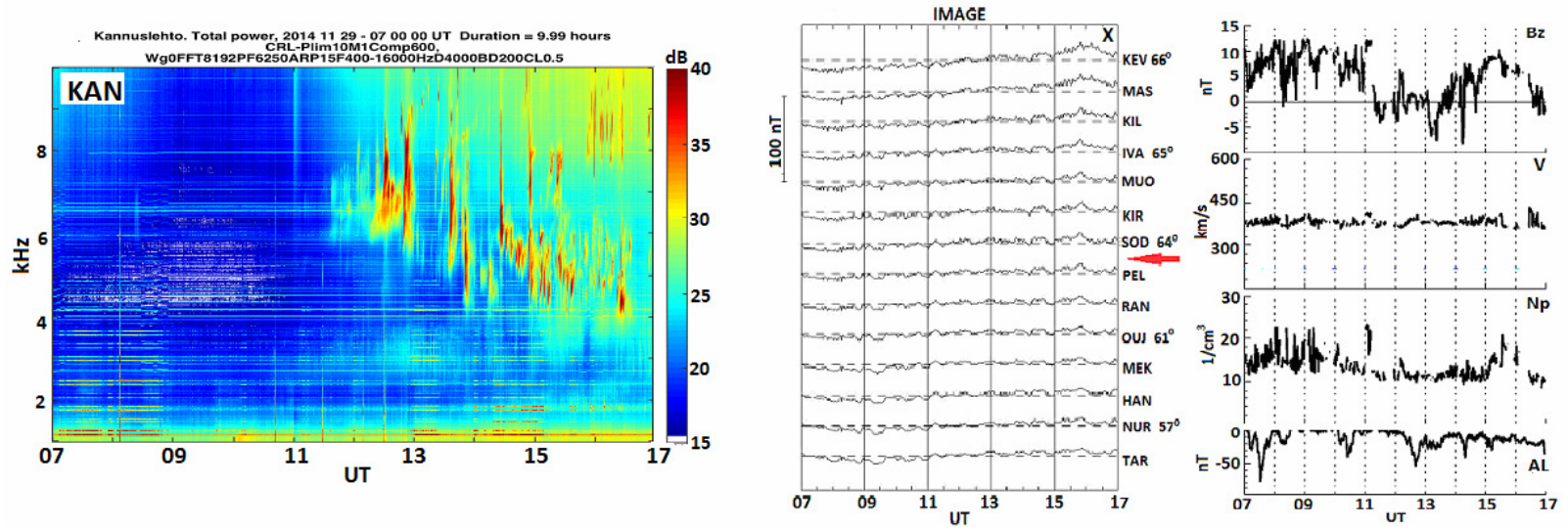

Fig. 4. (a) - The spectrogram of "bird-emissions" 29 November 2014; (b) - magnetic X components from IMAGE chain, and (c) parameters of the IMF and solar wind.

Very rich collection of different kind discrete VLF emissions was discovered after the filtering out the initial data of the wave records. An example of a raw unfiltered VLF one-hour spectrogram and the same data after filtering-out the sferics is plotted in Fig.1. It is seen that a new and very unusual spectral structure of signals above $4 \mathrm{kHz}$ appears after filtering. Several examples of the peculiar after-filtered VLF signals with various spectral forms are given in Manninen et al. (2016, 2017). Such signals were previously completely

unknown. Here we continue our study of the behaviour of these recently revealed VLF emissions more detailed concentrating the attention on the geomagnetic conditions favourable for their generation.

It is generally accepted that many magnetospheric waves are generated under solar wind and geomagnetic disturbances. However, here we present new and totally unexpected natural high-frequency electromagnetic VLF waves obtained after filtering out which occur during quiet space weather conditions. 


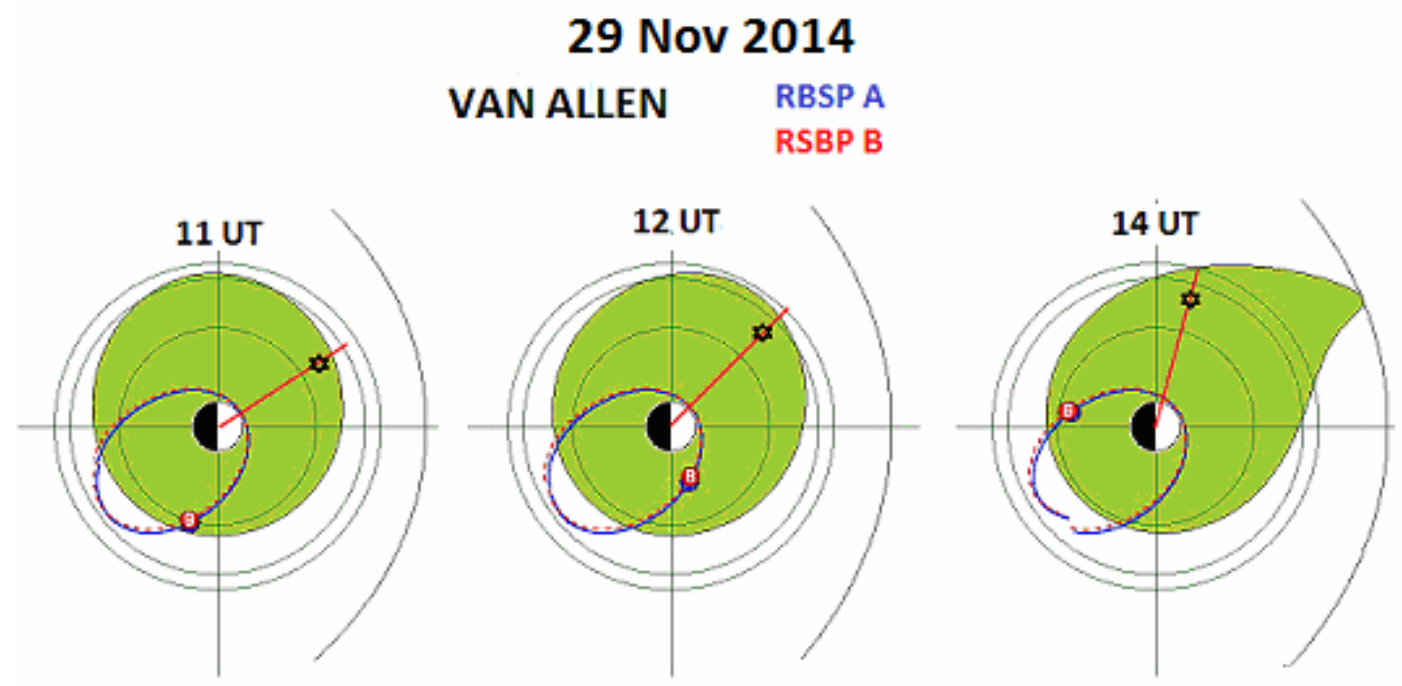

Fig. 5. The plasmapause location according to the Van Allen Probe data (http://enarc.space.swri.edu/PTP) . Relative location of KAN has been marked by a star and the red lines are showing the local time sector of KAN at the given time

\section{Dec 2014}
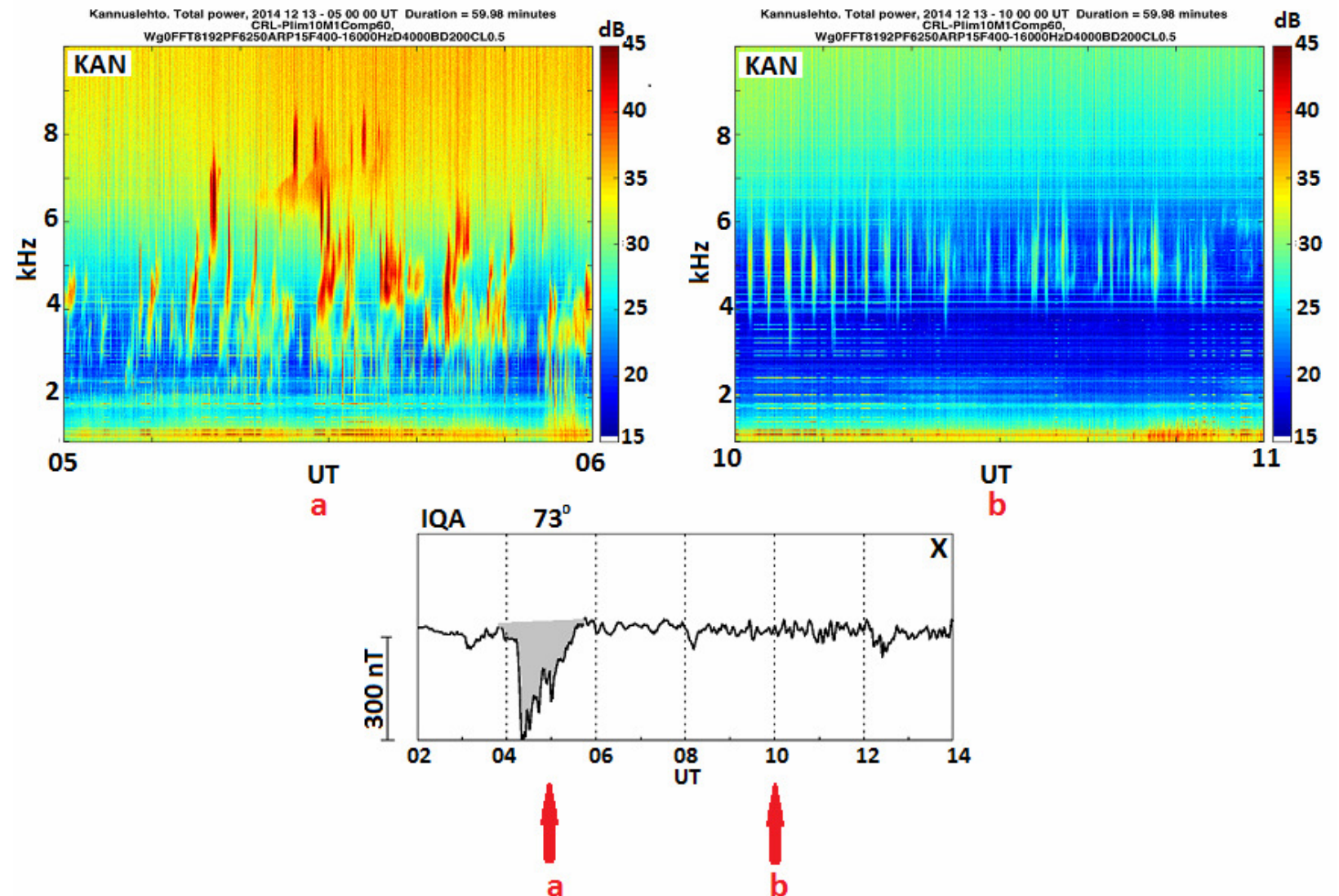

Fig. 6. One example of the "bird-emission" event with a change of the spectral shape observed on 13 December 2014.

\section{Observations}

Here we consider the behavior of the recently revealed new types of discrete VLF emissions at the frequencies above $4 \mathrm{kHz}$ recorded in KAN during several winter campaigns. Figure 2 displays the dynamic spectra of one example of the discussed events by two time scales. Very complex unusual spectral futures of the signals are seen. In the one-hour spectrograms, the signals look like sticks or wands, but their fine spectral structure is very peculiar and varies with time. Sometimes the signal shapes resemble flying birds as it is shown in Fig. 2 (b). When the recordings of these waves are played through a loudspeaker, they sound often like bird song; due to that, we call these high-frequency events "bird-emissions" (Manninen et al., 2016). Several events of the VLF "bird-emissions" with different spectral dynamics are shown in our previous papers (Manninen et al., 2016, 2017) where we presented as well the daily variations of these emissions. 


\section{Dec 2014}
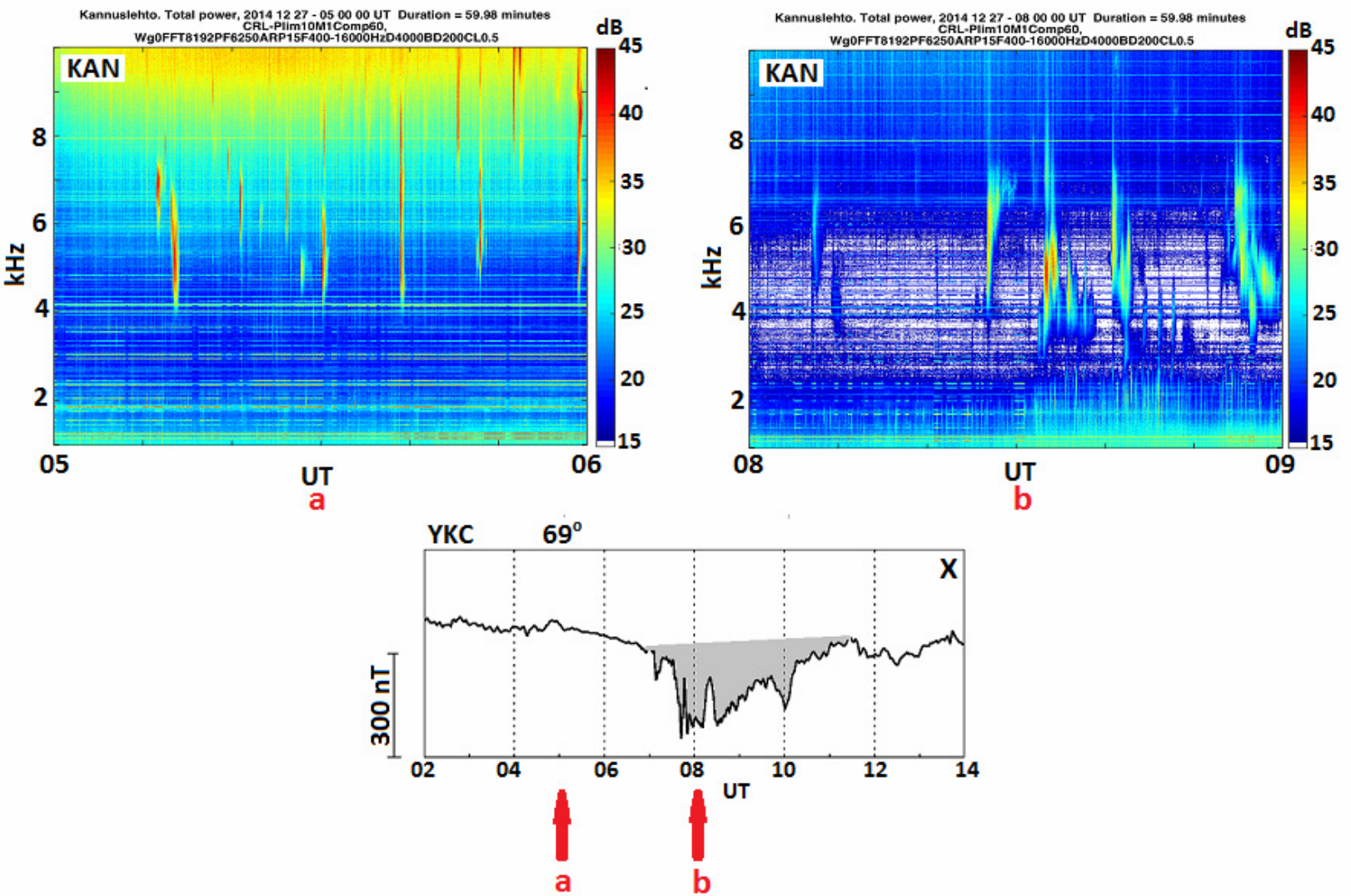

Fig.7. The same as in Fig. 6 but observed on 27 December 2014.

\section{Dec 2013}

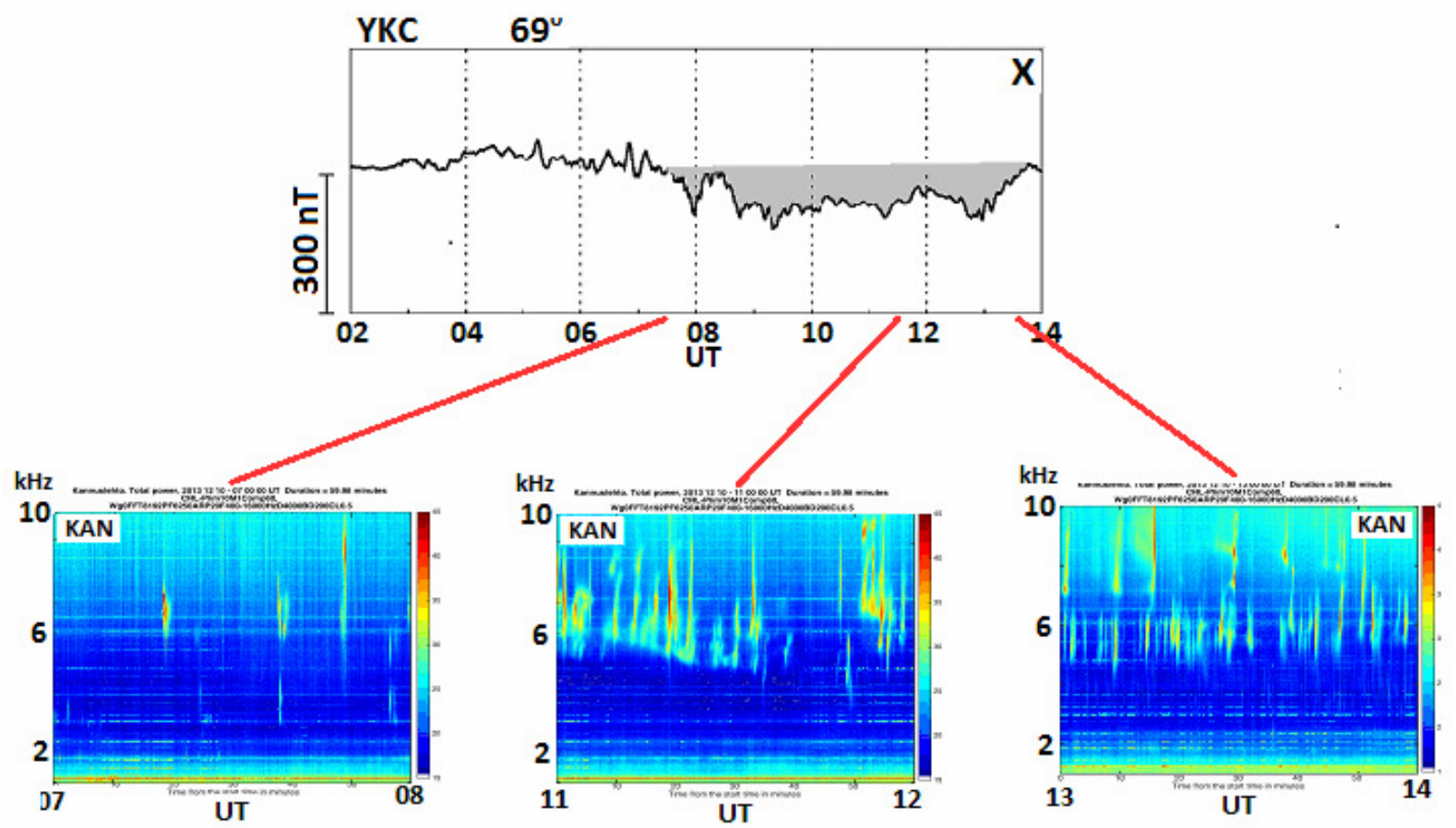

Fig. 8 An example of nighttime magnetic substorm at auroral latitudes observed at YKC station (MLT = UT-8) during the "bird emissions" recorded at KAN (MLT $=U T+3)$ on 10 December 2013. 
The "bird-emissions" are usually lasting for several hours and contain short burst-like structures at frequencies higher than $4 \mathrm{kHz}$, even up to $15 \mathrm{kHz}$ and, may be, more. The waves are typically right-hand polarized signals, suggesting that KAN was located in the vicinity of the wave exit area from the ionosphere. Different fine spectral structures of the discussed VLF

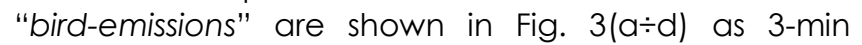
spectrograms on various days. Very often, the signal frequency increases with time as it is seen in Fig. 3(a, c, d). Sometimes a wide frequency band could generate simultaneously as is presented in Fig. 3b.

The considered VLF emissions were observed mostly in the day time, with the wide maximum of the occurrence around the local magnetic noon $(09 \div 15 \mathrm{MLT})$. They are common phenomena in the winter period. For example, in the winter campaign $2014 \div 2015$, these emissions were recorded in 52 out of 59 days of observation. A similar result was also

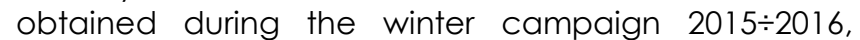
when the "bird-emissions" were recorded in 75 out of 91 days of observation. So, these emissions are typical winter daytime VLF phenomena

We found that typically, the several hours lasting "bird-emissions" are observed under quiet space weather conditions, i.e., the low values of the solar wind speed $\left(V_{s w}<400 \div 500 \mathrm{~km} / \mathrm{s}\right)$ and density $\left(\mathrm{Np}<5 \div 8 \mathrm{~cm}^{-3}\right)$. The geomagnetic activity was also low (Kp 1 $\div 2)$, and there were no significant substorms at the night-side $(\mathrm{AE}<150 \div 200 \mathrm{nT})$. We have to note that during the winter campaigns $2014 \div 2016$, strong geomagnetic storms were absent. However, the "birdemissions" occur a few days after periods of moderate geomagnetic disturbances.

One example of the space weather state during the VLF "bird-emissions" observed at KAN on 29 November 2014 at $11 \div 17$ UT $(K p=0 \div 1)$ is presented in Fig. 4. It was occurred four days after quite significant magnetic disturbances with $\mathrm{Kp}=5$. During these daytime emissions, there was no magnetic activity enhancement at the IMAGE magnetometer chain (Fig. $4 \mathrm{~b})$, and no substorms ( $A E<50 \mathrm{nT}$ ) at the night side. The solar wind velocity was low (Fig. 4C). The Van Allen Probe (Radiation Belt Storm Probes - RBSP) measuring the plasmapause location showed that, during this event, KAN (marked as star) was mapped inside the plasmasphere, but in the vicinity of the plasmapause (Fig. 5).

Two more "bird-emission" events are shown in Fig. 6 and 7 demonstrating that in course of the emission development, the spectral structure of the signals can change with time. The simultaneous night-side magnetograms are given in the bottom plots of Fig. 6 and 7. It is seen that the simple wave dynamic spectra (Fig. 6b and 7a) were observed under the absence of simultaneous night-side substorms. The more complicated wave spectral structures as in Fig. 6a and Fig. 7b were observed under small or moderate magnetic substorms at the auroral latitudes on the night-side of the Earth. In Figure 8, three intervals of the day-side "bird-emission" at KAN and the simultaneous night-side geomagnetic disturbance at auroral latitudes are presented on 10 December 2013. It is easily seen, that in the beginning of the substorm, the separated discrete VLF signals occurred at long intervals, and the events became more frequent during the development of the substorm.

The studied "bird-emissions" have been observed both in the presence and absence of typical lower frequency VLF emissions such as chorus, hiss, and quasi-periodic events. Typically, the "bird-emission" frequency increase with time, supporting the shift of the wave source location to the lower L-shells. However, the hiss-like frequency usually did not change in the same way. We could conclude that these signals were from different origins.

\section{Discussion}

It is generally accepted that the VLF whistler-mode waves are generated by the cyclotron instability of radiation belt energetic particles in the inner magnetosphere (e.g., Rycroft, 1972; Trakhtengerts and Rycroft, 2008). The frequency of excited waves is controlled by the equatorial electron gyrofrequency $f_{c e}$, which is proportional to the local magnetic field strength. Thus, the frequency shift of the emissions, shown in Fig. 3, indicates the special dynamics of the magnetospheric location of the wave generation region. We remember that the waves are guided to the ionosphere by magnetic field with the upper cutoff frequency at one half of the equatorial electron gyrofrequency fce (e.g., Carpenter, 1968).

The $f_{\text {ce }}$ value corresponding to the field line of KAN $(\mathrm{L} \sim 5.5)$ is $\sim 5.3 \mathrm{kHz}$. Thus, the wave propagated roughly along the KAN magnetic field line, can reach the ground only at $f \leq 2.6 \mathrm{kHz}$. Such waves are seen in Fig. 8 as strong hiss-like emissions in the bottom part of the spectrograms. Thus, the "bird-emissions", observed in KAN at frequencies above $4 \div 6 \mathrm{kHz}$, should be generated at lower L-shells where the equatorial electron gyrofrequency $f_{c e}$ is higher than $5.3 \mathrm{kHz}$.

Recently, Titova et al. (2015) reported simultaneous observations of the high-frequency QP emissions, showing similar spectral and frequency features between ground-based VLF receiver at KAN and Van Allen Probe spacecraft located above UK to SouthWest from KAN. The authors concluded that this QP event was generated inside the plasmasphere at much lower L-shells than KAN, but they did not discuss the propagation properties of these emissions. Strangeways et al. (1983) observed one-hop whistlers recorded at three high-latitude stations with the exitpoints located at $L \sim 6.4$. These whistlers were originated at much lower $L$-shells, namely, at $L \sim 2.8 \div 4.0$. This disparity was explained by the whistlers first following a field-aligned ducted path inside the plasmasphere, after being reflected by sporadic E layer in the lower ionosphere, following a sub-protonospheric path outside the plasmapause to higher latitudes.

\section{Summary}

Based on the VLF observations in Northern Finland at Kannuslehto (KAN, L $~ 5.5$, where $f_{\mathrm{ce}} \sim 5.3 \mathrm{kHz}$ ), we revealed new and totally unexpected natural VLF electromagnetic emissions at frequencies higher than $4 \mathrm{kHz}$, i.e., well above $f_{\mathrm{ce}} / 2$ at $\mathrm{L} \sim 5.5$. We call these VLF waves "bird-emissions".

These emissions are observed as right-hand polarized waves lasting for several hours in the daytime and containing short $(\sim 1 \div 3 \mathrm{~min})$ burst-like structures. 
It was found that the low solar wind and geomagnetic activity $\left(\mathrm{V}_{\mathrm{sw}}<400 \div 500 \mathrm{~km} / \mathrm{s}\right.$, $\mathrm{Np}<5 \div 8 \mathrm{~cm}^{-3}, \mathrm{AE}<150 \div 200 \mathrm{nT}$ ) are the favourable conditions for the "bird-emissions" generation and propagation. This finding allowes us to suppose that this new type of high-frequency VLF emissions could represent the self-oscillations of the magnetosphere maser discribed in (Trakhtengerts and Rycroft, 2008). The emissions appear after moderate geomagnetic disturbances or in the late recovery phase of geomagnetic storms.

We suppose that the "bird-emissions" are generated deep in the magnetosphere via the electron-cyclotron instability at much lower $L$ values than KAN (L 5.5). A small substorm develoment in the night sector could be a source of the electrons injected into the magnetosphere from the magnetotail and trapped into the Earth's radiation belts.

However, the details of the mechanism of the generation and propagation of these newly discovered VLF emissions remain unknown.

\section{Acknowledgements}

The research was supported by the Academy of Finland (grant no. 287988 for N.K. and L.G.), and the University of Oulu (grant for M.J.R.). We are grateful to the technical staff of SGO for their valuable assistance in organising the field campaigns. The work of N.K. was partly supported by the Program No 28 of the Presidium of the Russian Academy of Sciences (RAS).

\section{References}

Carpenter, D.L. (1968). Ducted whistler-mode propagation in the magnetosphere: A half-gyrofrequency upper intensity cut-off and some associated wave growth phenomena, J. Geophys. Res., 73, 2919-2928.

Helliwell, R.A. (1965). Whistler and related ionospheric phenomena. -Stanford: Stanford Univ. Press.

Manninen, J. (2005). Some aspects of ELF-VLF emissions in geophysical research, Sodankylä Geophys. Obs. Publ. no. 98, Oulu Univ., 2005. http://www.sgo.fi/Publications/SGO/thesis/ManninenJyrki. pdf.

Manninen, J., T. Turunen, N. Kleimenova, M. Rycroft, L Gromova, and I. Sirviö (2016). Unusually high frequency natural VLF radio emissions observed during daytime in Northern Finland. Environ. Res. Lett., 11, 124006, doi:10.1088/17489326/11/12/124006

Manninen, J., T. Turunen, N.G. Kleimenova, L.I. Gromova, and A.E. Kozlovsky (2017). A new type of daytime high-frequency VLF emissions at auroral latitudes ("Bird emissions"). Geomagn. Aeron., 57, no. 1, 36-44, doi:10.1134/S0016793217010091.

Ohya, H., K. Shiokawa, and Y. Miyoshi (2015). Daytime tweek atmospherics, J. Geophys. Res.: Space Phys., 2015, vol. 120, no. 1 , pp. 654-665, doi 10.1002/2014JA020375

Rycroft, M.J. (1972). VLF emissions in the magnetosphere, Radio Sci., vol. 7, pp. 811-830. doi 10.1029/RS007i008p00811

Smith, R.L., R.A. Helliwell, and I.W.A. Yabroff (1960). Theory of trapping of whistlers in field-aligned columns of enhanced ionisation, J. Geophys. Res., vol. 65, pp. 1-20.

Strangeways H.J., M.A. Madden, and M.J. Rycroft (1983). High latitude observations of whistlers using three spaced goniometer receivers. J. Atmos. Terr. Phys. Vol. 45, No 6, pp. 387-399.

Titova, E.E., B.V. Kozelov, A.G. Demekhov, J. Manninen, O. Santolik, C.A. Kletzing, and G. Reeves (2015). Identification of the source of quasiperiodic VLF emissions using groundbased and Van Allen Probes satellite observations, Geophys. Res. Lett., vol. 42, pp. 6137-6145, doi:10.1002/2015GL064911.

Trakhtengerts, V.Y. and M.J. Rycroft (2008). Whistler and Alfven Mode Cyclotron Masers in Space. - Cambridge. U.K.: Cambridge Univ. Press. 